\title{
High Tibial Osteotomy for Genu Varum in Adults: Do Proprietary Implants Limit the Quality of Correction?
}

\author{
Solomin $\mathrm{LN}^{1}$, Chugaev DV ${ }^{2}$, Filippova $\mathrm{AV}^{3}$, Kulesh PN ${ }^{4}$
}

\begin{abstract}
The surgical technique of proximal tibial osteotomy for genu varum in adults has evolved from a procedure using closing wedges of estimated sizes with staple fixation in the 1960s to using standard trauma internal fixation implants and, more recently, to gradual correction with software-guided hexapod external fixators. In the last two decades, implant manufacturers have also produced anatomical implants specific for such corrective osteotomies. This study evaluates the limits of using such proprietary implants for proximal tibial osteotomy in genu varum. Materials and methods: Scanograms (teleradiograms) of lower limbs of a patient were used to derive skiagrams (two-dimensional bony outlines of the extremities). From these, two-dimensional and three-dimensional models of varus deformities of the tibia with different values of mechanical medial proximal tibial angle ( $\mathrm{mMPTA}$, from $85^{\circ}$ to $40^{\circ}$ ) were created. An analysis of the created deformity was carried out and a simulation for surgical correction was performed using an open wedge high tibial osteotomy with fixation using a proprietary (Tomofix, Synthes) implant. In addition, a 3D simulation technique was used to check the accuracy of the results obtained from the 2D simulation.

Results:

- In cases of mMPTA $\geq 80^{\circ}$ with localisation of the apex of varus deformity at the level of the knee joint line, the standard technique used with the proprietary medial tibial plate produces good results.

- In cases of $\mathrm{mMPTA} \leq 70^{\circ}$, fixation of the osteotomised fragments by the proprietary medial plate is poor owing to the anatomical contours of the implant. In these cases, a different type of osteosynthesis is needed.

- In cases of $\mathrm{MMPTA} \leq 70^{\circ}$, the distance between the lower edge of the bone plate and the medial surface of the tibia after a proximal tibial osteotomy exceeds $11 \mathrm{~mm}$ and will result in unacceptable soft tissue tension around the implant.

- Mechanical axis deviation to the Fujisawa point produces MMPTA values outside the reference range of normal values.

Conclusion: An osteotomy of the proximal tibia using a prescribed technique linked to a proprietary implant achieves good results only if performed within a certain range of deformity values. Pronounced varus deformities require a fundamentally different approach. This study reveals that surgeons undertaking corrective proximal tibial osteotomies for genu varum need to perform a comprehensive analysis of the deformity to allow for appropriate selection of patients. This will enable a consideration of the size and other characteristics of the deformity that will reduce the technical complications that may arise if the correction was performed using the recommended technique linked to a proprietary implant.

Keywords: Deformity correction, Deformity planning, Genu varum, Limb deformity, Limb reconstruction, Locking plate, Osteotomy, Varus deformity knee.

Strategies in Trauma and Limb Reconstruction (2020): 10.5005/jp-journals-10080-1449
\end{abstract}

\section{INTRODUCTION}

Early studies reporting the results of the realignment osteotomies for deforming arthrosis of the knee joint were published in 1960s.' At that time, it was a declared surgical objective to "make the knee straight" and restore the joint line of the knee to be parallel to the floor. ${ }^{2}$ An evolution of the technique came with improvement of implants for osteosynthesis and a widening of indications for this treatment strategy. Improvements in imaging technology together with incorporation of computing, navigation, and the use of prototyping and other advances led to further applicability of this surgical procedure with better predictability of the outcome. ${ }^{3-8}$ These gains made the entire procedure of corrective osteotomy safer for patients. ${ }^{9-11}$

In a parallel development, joint replacement with endoprostheses established itself as an effective method for treatment of knee arthrosis. This was responsible for a reduced interest in corrective realignment osteotomies as a means of treatment. Long-term follow-up reports revealed, however, a number of technical and clinical limitations in endoprosthetic replacements for knee arthrosis especially when used for young patients. There were matters as polyethylene wear, aseptic loosening of components, and infection

\footnotetext{
1,2National Medical Research Center of Traumatology and Orthopedics, Saint Petersburg State University, Saint Petersburg, Russia, Russian Federation

${ }^{3}$ Department Saint Petersburg State University, Saint Petersburg, Russia, Russian Federation

${ }^{4}$ St. George City Hospital, Saint Petersburg, Russia, Russian Federation Corresponding Author: Chugaev DV, National Medical Research Center of Traumatology and Orthopedics, Saint Petersburg, Russia, Russian Federation, Phone: +7 89213947459, e-mail: dr.chugaev@ gmail.com
}

How to cite this article: Solomin LN, Chugaev DV, Filippova AV, et al. High Tibial Osteotomy for Genu Varum in Adults: Do Proprietary Implants Limit the Quality of Correction? Strategies Trauma Limb Reconstr 2020;15(1):13-22.

Source of support: Nil

Conflict of interest: None

that produced a need for a new subspecialty-that of secondary and multiple revision arthroplasty. Added to this were some reports of functional results of total replacement of the knee joint that 
High Tibial Osteotomy for Genu Varum in Adults: Do Proprietary Implants Limit the Quality of Correction?

were not ideal. ${ }^{12,13}$ This, and the preference of younger patients to preserve their native knee joints and a desire to maintain a high level of physical activity, restored the role of corrective realignment osteotomies as a treatment option. This resurgence led orthopaedic surgeons to improving the technical aspects of this procedure and adapting it to the modern patient. ${ }^{14}$

A fundamental concept underpinning corrective realignment surgery of the lower limb, particularly in cases of deformity from gonarthrosis, is the mechanical axis of the limb. The concept of "limb axis" was first introduced into orthopaedic practice by Polish surgeon Mikulicz-Radecki. He identified that, in patients without frontal deformities of the lower limb, the line connecting the hip and the ankle joints passed through the centre of the knee joint and considered this reference point a key for successful deformity correction. ${ }^{15}$ Fujisawa investigated practical aspects of the realignment tibial osteotomy in the treatment of end-stage medial gonarthrosis. According to his postoperative arthroscopic findings, corrective tibial osteotomy resulted in the regression of degenerative changes in the knee joint when the mechanical axis was re-aligned to pass through a point $30-40 \%$ lateral to the middle of the knee. ${ }^{5}$ Additionally, further understanding of deformity and malalignment was possible through work by Paley who summarised and systematised the findings of his predecessors ${ }^{16,17}$ and published an approach to preoperative planning and assessing the results of corrective osteotomies of the lower limb by using an atlas of reference angles and lines.

In the last decade the introduction of corrective osteotomies in the proximal tibia using prescribed techniques linked to proprietary implants have emerged. These techniques carry a strong association with the implant promoted by the manufacturers and will now be referred to as 'proprietary' proximal tibial osteotomies. The aim behind these proprietary procedures is to simplify the intervention such that general orthopaedic surgeons are capable of it. ${ }^{5,6,8}$ The simplification extends such that the site, type and degree of osteotomy is dictated by the process of adhering to the technique described by the manufacturer rather than the individual characteristics of the deformity. This assumes that accurate adherence to these guidelines when performing the corrective tibial osteotomy is sufficient for success, irrespective of the degree of genu varum associated with the monolateral knee arthrosis.

\section{Aim of the Study}

This study evaluates the possible outcomes from simulating an open wedge proximal tibial osteotomy in patients with different values of the mechanical medial proximal tibial angle (mMPTA) using 2D (skiagrams) and 3D models of the lower extremities.

\section{Materials and Methods}

Frontal scanograms (teleradiograms) of the lower extremities of an adult $180 \mathrm{~cm}$ man with no history of orthopaedic pathology were used. Graphic outlines (skiagrams) of the lower limb bones were created on drawing paper using a negatoscope. These skiagrams were scaled proportionally to the A4 format in Adobe Photoshop. This graphic sample was used to create models reflecting varus deformities of the tibia with different MMPTA values from $85^{\circ}$ to $40^{\circ}$, in steps of $5^{\circ}$ for deformities with $\mathrm{mMPTA} 85^{\circ}$ and $80^{\circ}$ and then in steps of $10^{\circ}$ (Fig. 1).
Simulation was performed under the condition that the apex of varus deformity (the centre of rotation of angulation, CORA) was localised at the level of the knee joint line reflecting the most common clinical situation of a patient with monolateral gonarthrosis. In this clinical problem the deformity arises predominantly from an intra-articular change and there are no pathological changes to the reference values of tibial metaphysis and diaphysis. These models were taken for preoperative planning using Adobe Photoshop as an image editor to simulate open wedge a proximal tibial osteotomy with subsequent plate fixation.

For every graphic model two variants of tibial correction were planned: firstly, up to the "ideal" value in which the resultant mechanical axis passes through the centre of the knee joint; and secondly, with an overcorrection in which the mechanical axis passes through the Fujisawa point, i.e., with lateral deviation of the mechanical axis up to $10 \mathrm{~mm}$. After simulation was performed, the values of MMPTA and lateral distal tibial angle (LDTA) were measured.

Thus, the mechanical axis of the leg and its deviation, CORA, MPTA, and LDTA were the key parameters that were measured on the graphic models. These were chosen to reflect the degree of the 'created' deformity of the lower limb and were most likely to change by the simulated correction.

To confirm the accuracy of the measurements using 2D simulation, we used a 3D simulation technique based on computed tomography scans of the legs of the same healthy man whose scanograms were used to source the skiagrams earlier. The measured values of the key parameters were as follows: femur length $-43 \mathrm{~cm}$, tibia length-35 cm, lateral distal femoral angle $($ LDFA $)=88^{\circ}$, MPTA $=89^{\circ}$, LDTA $=89^{\circ}$.

Using 3D Slicer, a Cinema 4D software, virtual graphic models of the right lower limb with a varus deformity of tibia, with the values of MPTA in the range specified above (from $89^{\circ}$ to $40^{\circ}$ ), were created. The CORA of the varus deformity was placed at the level of the knee joint line, as before, in all cases. Following a virtual assessment of the reference lines and angles and evaluation from different projections (using perspectives right, left, top, front), the osteotomy planes were mapped out and modelling of a corrective open-wedge osteotomy was performed. The distal tibia segment was moved following the position of a rotation axis placed to correspond to a lateral hinge. Two simulations were performed; to the neutral (normal) position of the mechanical axis and with overcorrection to the Fujisawa point.

After performing the simulation, the possibility of fixing bone fragments by an anatomical medial bone plate was checked. Based on existing templates of proprietary implants used for proximal tibial corrective osteotomies, the plate was taken as having the following properties: a T-shape, angular stability of screws in direction of insertion, and anatomical correspondence of the plate to the medial surface of the proximal tibia. The congruence of this implant to the bone fragments of the model was evaluated in various projections (perspective, right, left, top, front) by varying the transparency of the virtual image from 0 to $100 \%$. Data from 2D and 3D simulations allowed for the results to be compared. In order to obtain objective criteria for the possibility of performing osteosynthesis with the use of a bone plate, we measured and evaluated the distance between the lower edge of the plate and 

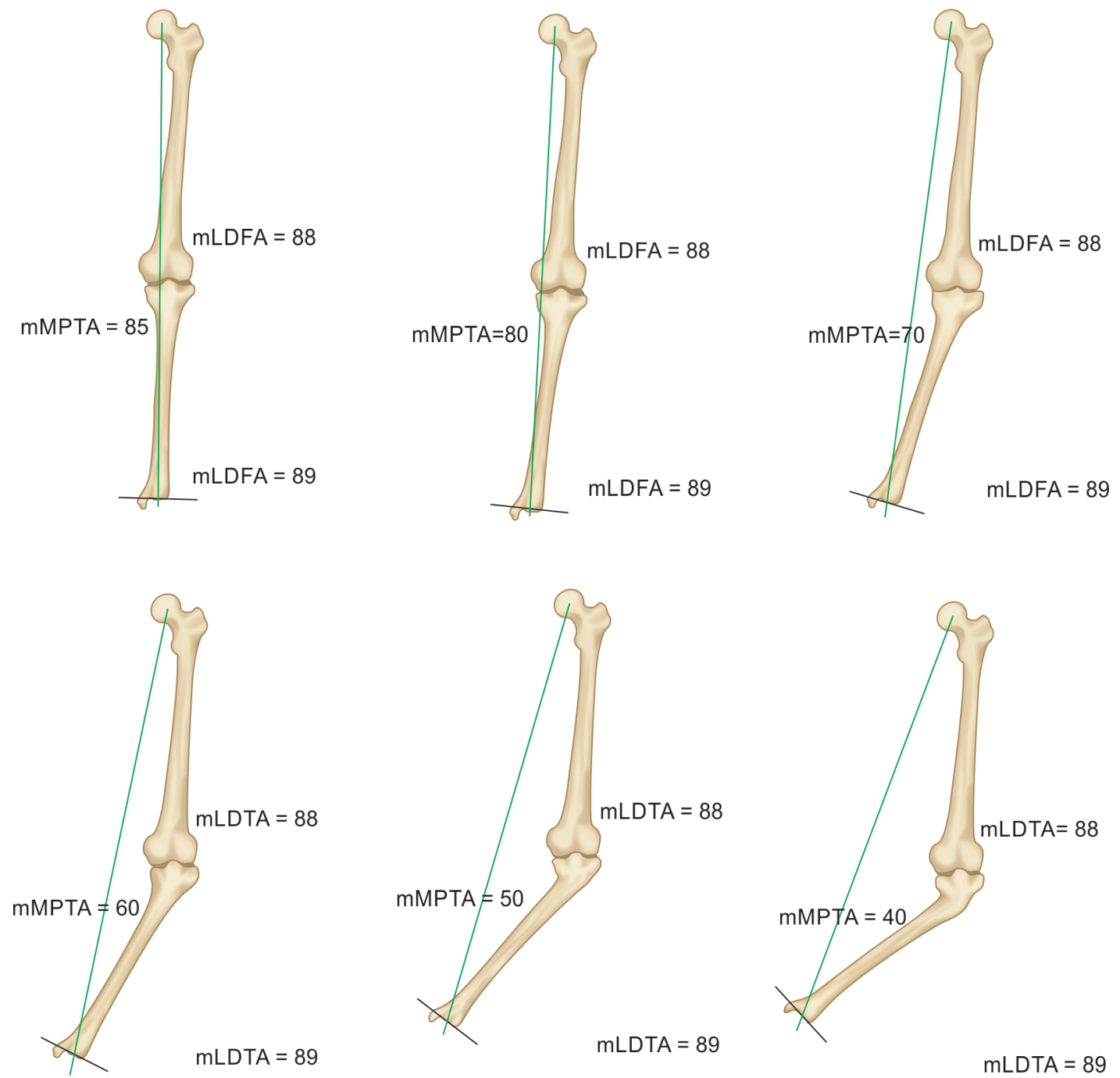

Fig. 1: Graphic models varus deformities of the lower limb with different values of mechanical medial proximal tibial angle

the medial surface of the tibia using digital methods and scaling to actual size.

\section{Results}

The 2D graphic and 3D models of different degrees of varus deformity of the lower extremity were used as the sample. For every model, preoperative planning was performed by simulating a corrective open-wedge proximal tibial osteotomy until two endpoints: to the "zero" point ( in which the mechanical axis passes through the centre of the intercondylar eminence) and to the point of overcorrection (in which the mechanical axis passes through the Fujisawa point).

When deformities with an MPTA $=85^{\circ}$ and MPTA $=80^{\circ}$ were corrected to produce a mechanical axis deviation (MAD) of zero, the values of the reference angles (MPTA, LDTA) do not exceed the limits of normal values. If overcorrection was simulated, there is an increase in the MPTA of up to $92^{\circ}$. Inserting an anatomical plate is possible without anticipated technical difficulties in these cases of correction of deformities with an MPTA $=85^{\circ}$ (Fig. 2) and MPTA $=80^{\circ}$
(Fig. 3). In both models, the distance between the lower edge of the plate and the medial surface of the tibia amounted to $0 \mathrm{~mm}$.

In correction of a deformity where the MPTA $=70^{\circ}$ is improved to a $M A D=0$, the values of the reference angles do not exceed the limits of normal values. Overcorrection also results in an increase in MPTA to $92^{\circ}$. However, due to the size of correction needed (despite the rotation axis of the correction being in the same position as previous cases), plating becomes impossible due to the incongruence of the plate and the osteotomised proximal part of the tibia. With the mechanical axis passing through the Fujisawa point, it is also impossible to fix the bone fragments in the position of the achieved correction by using a medial plate (Fig. 4).

In correcting deformities with MPTA values from $70^{\circ}$ to $40^{\circ}$, the observed trend continues: in cases of reaching a $M A D=0$ the values of reference angles do not exceed the limits of normal values whereas the movement of MAD to the Fujisawa point leads to an increase in MPTA up to $92^{\circ}$. In case of correcting deformities with MPTA $=60^{\circ}$ (Fig. 5), MPTA $=50^{\circ}$ (Fig. 6) and MPTA $=40^{\circ}$ (Fig. 7) the effective lateral translation of the shaft of the tibia renders successful fixation of the bone fragments with a proprietary 


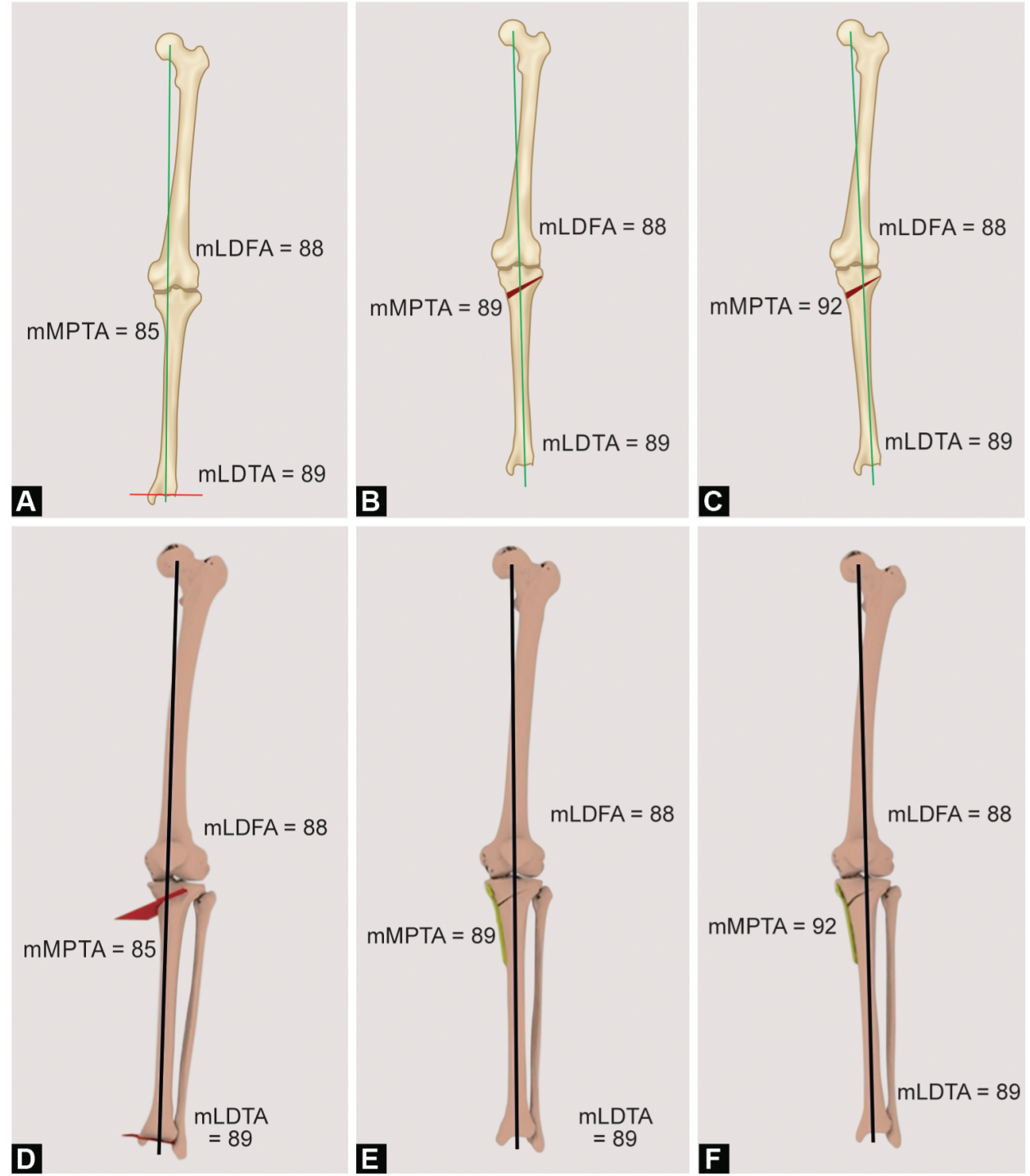

Figs $2 \mathrm{~A}$ to F: Models $\mathrm{A}$ to $\mathrm{C}$ demonstrate the technical possibility of deformity correction and plate fixation of bone fragments with $M P T A=85^{\circ}: 2 \mathrm{D}$ simulation ( $A$-initial deformity, B - correction to the neutral position of the mechanical axis, C-overcorrection). Models $\mathrm{D}$ to $\mathrm{F}$ show the technical possibility of deformity correction and plate fixation of bone fragments with MPTA $=85^{\circ}$ : 3D simulation (D-initial deformity, E-correction to the neutral position of the mechanical axis, F-overcorrection)

anatomical medial plate impossible. In this series of models, we observed the following changes in the distance between the lower edge of the bone plate and the medial surface of the tibia: $11 \mathrm{~mm}$ for MPTA $70^{\circ}, 17 \mathrm{~mm}$ for MPTA $60^{\circ}, 27 \mathrm{~mm}$ for MPTA $50^{\circ}$, and 30 $\mathrm{mm}$ for MPTA $40^{\circ}$. The plate would create a significant prominence beneath the medial subcutaneous border of the tibia that would be intolerable to the patient at the smaller distances and impossible to fix at the larger ones.

\section{Discussion}

There are several ways for planning corrective osteotomies for patients with limb deformities. Historically, simulations made on analogue skiagrams-outline tracings of the bone contours from the scanograms-were technically easy to model and use for singleplane osteotomy planning. ${ }^{14,16}$ The logical and technical evolution of this was use of graphics software to form single-plane graphic models from digital pictures of scanograms and to perform the planning and simulation of the correction. ${ }^{18,19}$

Single-plane skiagrams are unable to reflect the true spatial characteristics of a bone deformity. A modern approach will involve the use of 3D modelling; in addition to high-quality visualsation of the deformity, it also allows for accurate measurement of the key parameters, precise simulation of different variants of mechanical axis correction, and choice of the most effective method for fixation. ${ }^{20-23}$ 

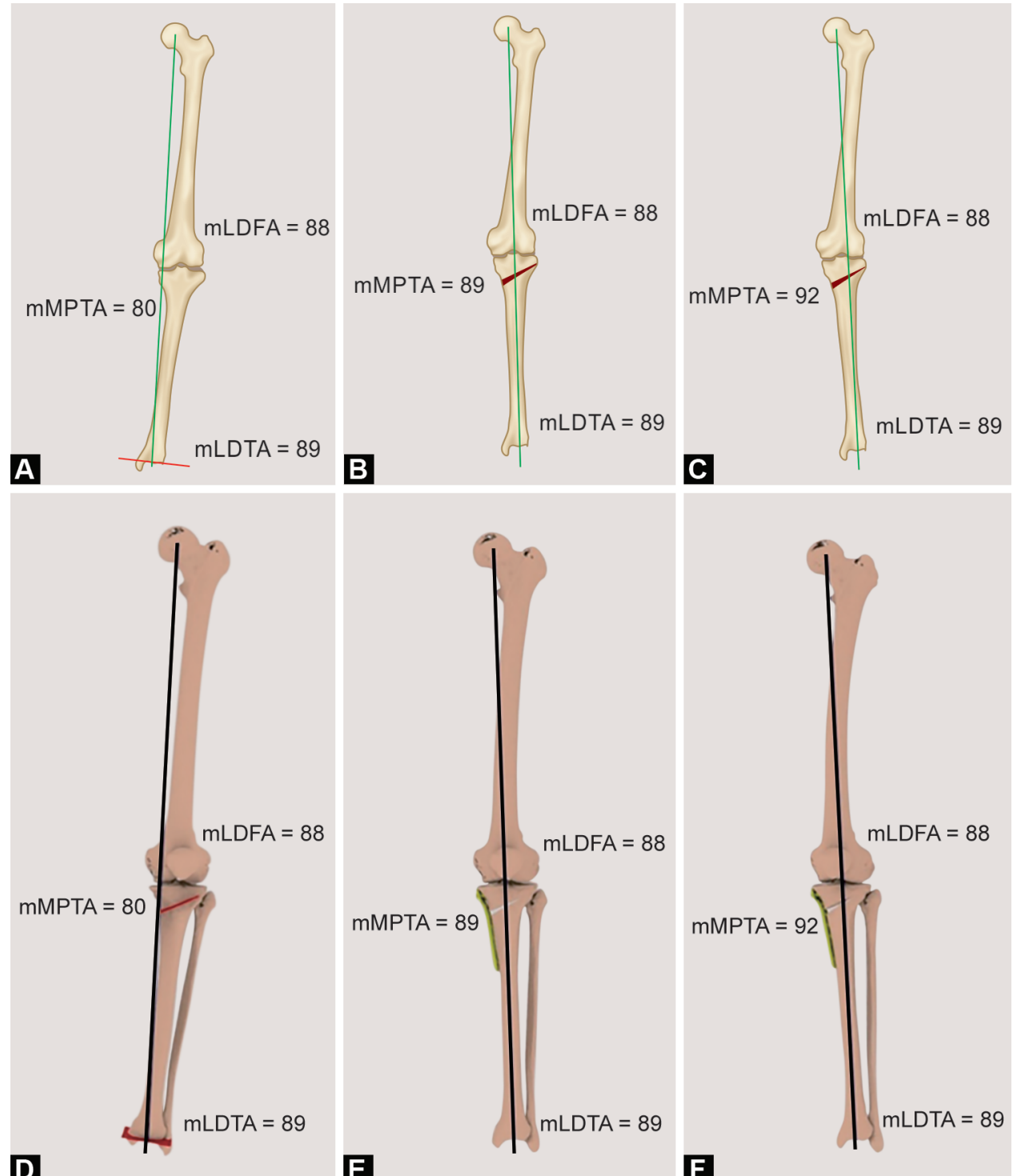

Figs $3 \mathrm{~A}$ to F: Models A to $\mathrm{C}$ demonstrate the technical possibility of deformity correction and plate fixation of bone fragments with $M P T A=80^{\circ}: 2 \mathrm{D}$ simulation ( $A$-initial deformity, B - correction to the neutral position of the mechanical axis, C-overcorrection). Models $D$ to $F$ show the technical possibility of deformity correction and plate fixation of bone fragments with MPTA $=80^{\circ}: 3 \mathrm{D}$ simulation ( $\mathrm{D}$-initial deformity, E-correction to the neutral position of the mechanical axis, F-overcorrection)

Key questions for the effective planning of a corrective tibial osteotomy are:

- Does the patient have a bone deformity?

- At what level is the CORA (centre of rotation angulation) located?

- What degree of deformity is present?

Paley et al. published reference lines and angles of the lower limb and formulated basic concepts of deformity analysis. The CORA method was described as were "osteotomy rules". In the first rule of osteotomy, the optimal level for the osteotomy is at the level of CORA. If this is deemed impossible or inappropriate technically, the greater the distance the osteotomy level is from the CORA level, the greater the translation (transverse movement) of the distal bone fragment will become in order for the proximal and distal axes of the relevant bone segments to become aligned (the second osteotomy rule). Therefore, from the CORA method of deformity analysis, a proximal tibial osteotomy for medial compartment gonarthrosis is a particular case where the CORA is close to the knee joint ${ }^{16}$ and the second rule becomes mandatory.

Paradoxically, these basic osteotomy rules are ignored in proximal tibial osteotomies carried out with proprietary implants. A possible explanation is that designers of the implant and technique prioritise favourable conditions for fixation of the osteotomised bone fragments rather than adhering to the rules of osteotomy. Consequently, what becomes a matter of primary concern is whether the size and position of the bone fragments after osteotomy is sufficient to achieve stable fixation using the proprietary implant. ${ }^{8,24-26}$

If a significant periarticular bone deformity requires correction, and if the CORA resolves to the joint level, then performing an osteotomy using the second rule will create problems of adequate 

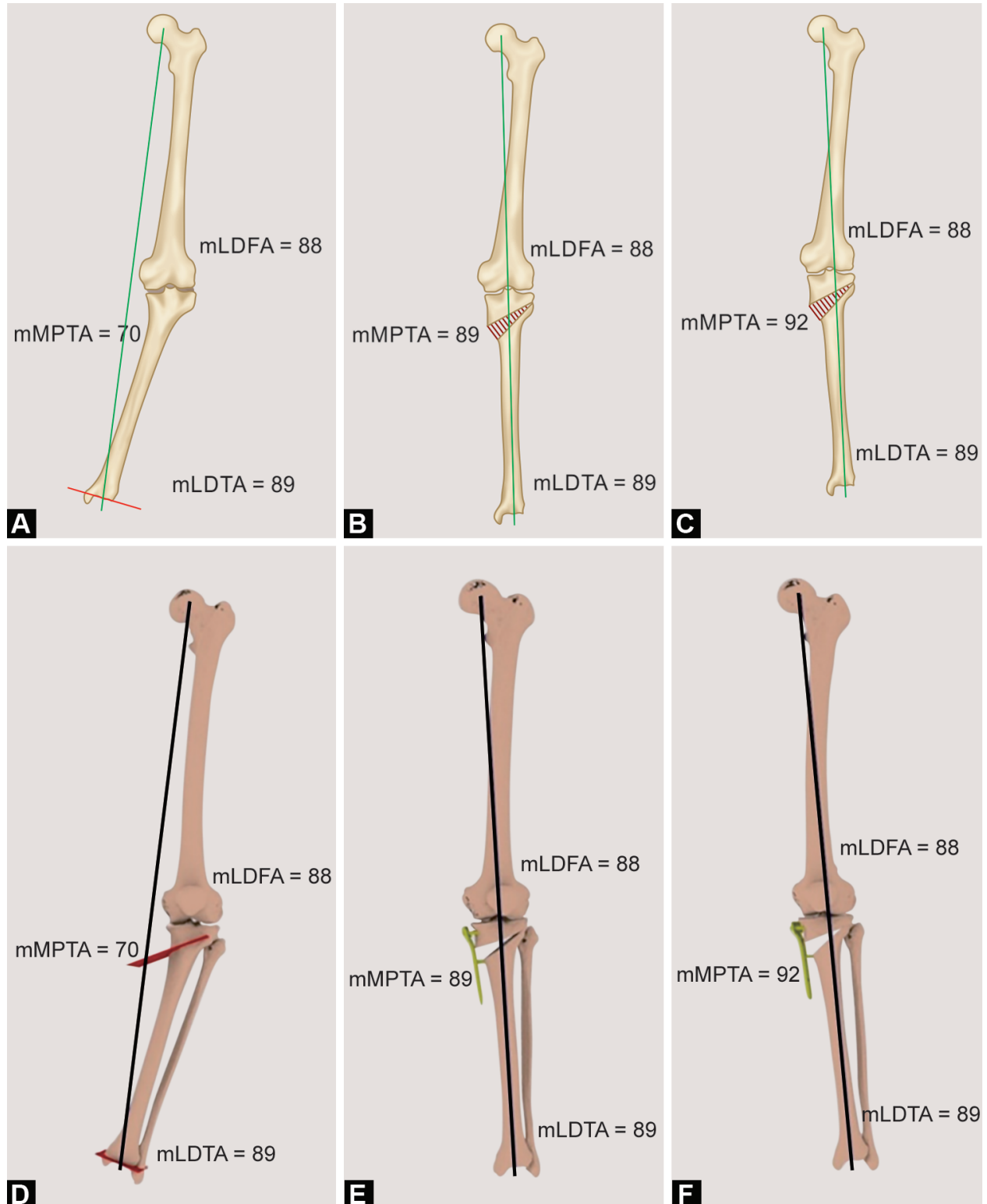

Figs 4A to F: Models $A$ to $C$ demonstrate the technical impossibility of deformity correction and plate fixation of bone fragments with MPTA $=$ $70^{\circ}: 2 \mathrm{D}$ simulation (A-initial deformity, B-correction to the neutral position of the mechanical axis, C-overcorrection). Models $\mathrm{D}$ to $\mathrm{F}$ show the technical impossibility of deformity correction and plate fixation of bone fragments with MPTA $=70^{\circ}: 3 D$ simulation (D-initial deformity, $\mathrm{E}$-correction to the neutral position of the mechanical axis, F-overcorrection)

coaptation and fixation by the implant because of translation of the distal bone segment. ${ }^{17}$ This scenario is met because this osteotomy technique involves rotation of the distal bone segment around a point on the preserved lateral cortex, the so-called "lateral hinge". A fracture of the lateral cortex is considered a complication because use of a medial bone plate in this situation does not ensure the necessary stability of the bone fragments. ${ }^{27-29}$ Whilst use of such a "standardised" osteotomy technique has advantages in being simpler to learn and offers repeatability, it is successful in correcting deformities within a certain range only.

In recent years, the literature has reported on double level osteotomies-distal femoral and proximal tibial osteotomies for pronounced varus deformity of the knee joint where using only a high tibial osteotomy can be inadequate or complicated technically. We have shown here the large size of the wedge and the difficulties with positioning the medial tibial plate make this procedure impractical. In this case, performing an osteotomy at another level would reduce the size of the wedge and help to achieve the required degree of the deviation of the mechanical axis. Thus, in the study by Schröter et al. we see that the authors perform a femoral osteotomy in a group of patients even though some of the patients have preoperative mLDFA values within the reference range. ${ }^{30}$ This approach of correcting a deformity of a bone by creating a deformity of another bone 

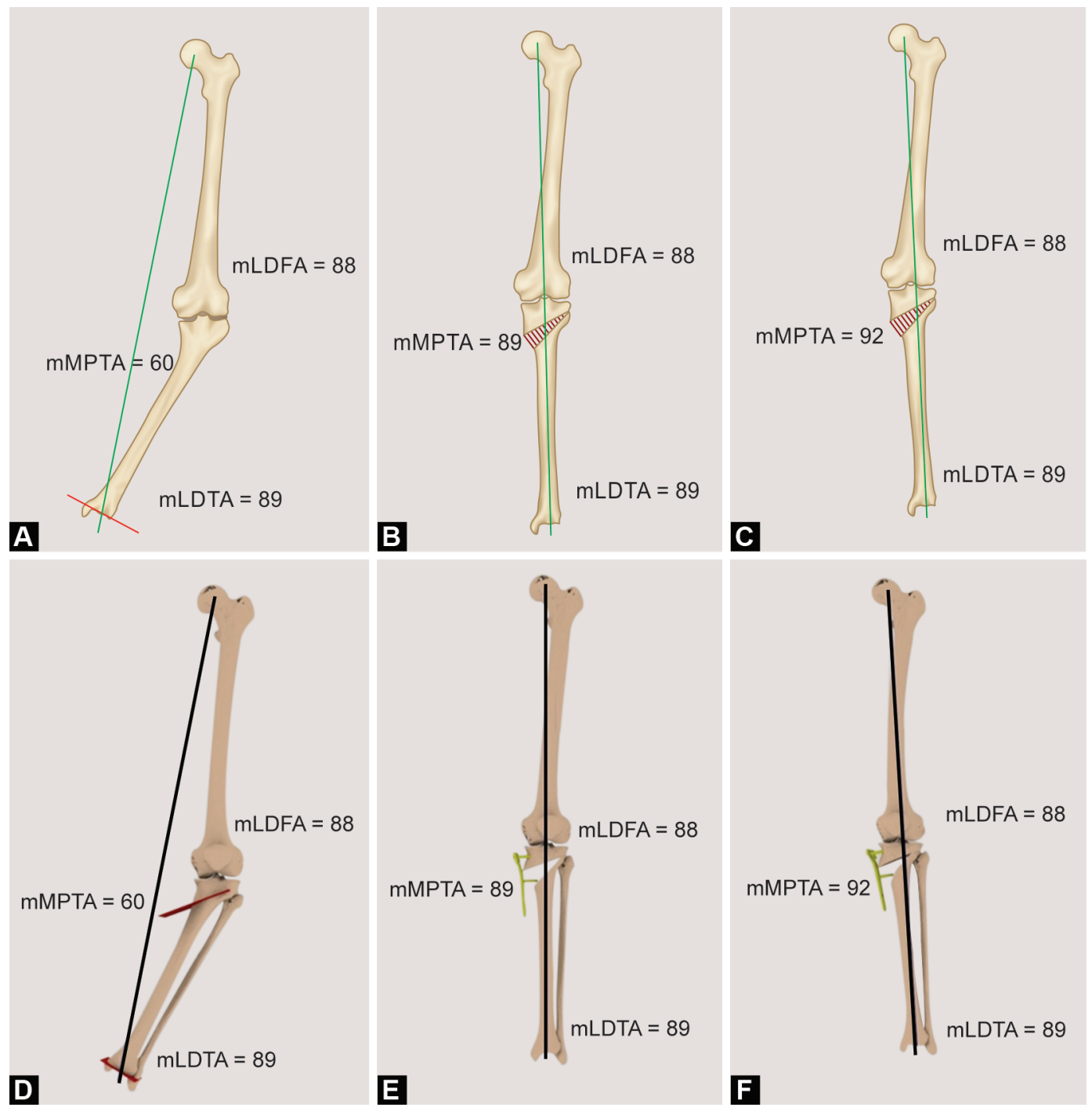

Figs $5 \mathrm{~A}$ to $\mathrm{F}$ : Models $\mathrm{A}$ to $\mathrm{C}$ demonstrate the technical impossibility of deformity correction and plate fixation of bone fragments with MPTA $=$ $60^{\circ}$ : $2 \mathrm{D}$ simulation (A-initial deformity, B-correction to the neutral position of the mechanical axis, $\mathrm{C}$ - overcorrection). Models $\mathrm{D}$ to $\mathrm{F}$ show the technical impossibility of deformity correction and plate fixation of bone fragments with MPTA $=60^{\circ}$ : $3 \mathrm{D}$ simulation (D—initial deformity, $\mathrm{E}$-correction to the neutral position of the mechanical axis, $\mathrm{F}$-overcorrection)

should not be undertaken as a preferred choice as it may lead to an oblique joint line and contradicts the principles of deformity correction. ${ }^{17}$ In our study we have shown that if the degree of the deformity of the proximal tibia is small (mMPTA $<80$ ), it allows for correction using a medial bone plate comfortably. Larger degrees of the deformity are not catered well by this technique and other approaches should be used, but not those involving the production of a "correction" in normal bones where the reference angles are within the normal range. Our study shows clearly that pronounced varus deformities (MPTA values of $70^{\circ}$ and less) require a fundamentally different approach for correction in regard to planning, technique and choice of stabilisation device post-osteotomy.

\section{Limitations of the Study}

This study evaluated the applicability and limits of corrective osteotomies of the proximal tibia performed using techniques described for proprietary implants. The study did not use original radiographs of patients with genu varum treated by proximal tibial osteotomy. The design of this study was to answer if it was possible to use such proprietary techniques for various MPTA values; using actual radiographs of patients with this range of values would not have been practical.

In order to standardise the study and to simplify the evaluation of the obtained data, it was assumed that the femur had no deformities. In the graphical analysis of 2D and 3D models with 


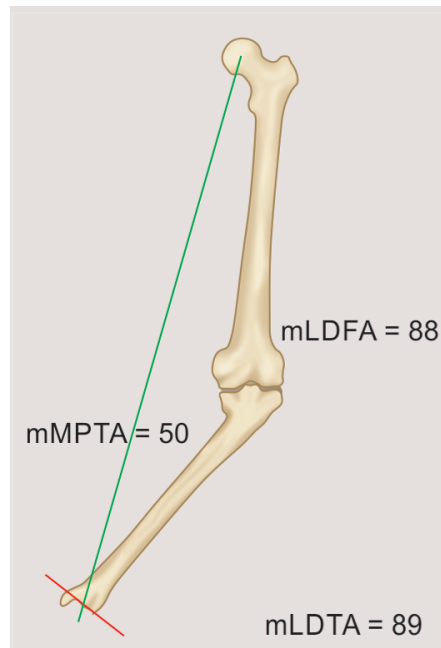

A

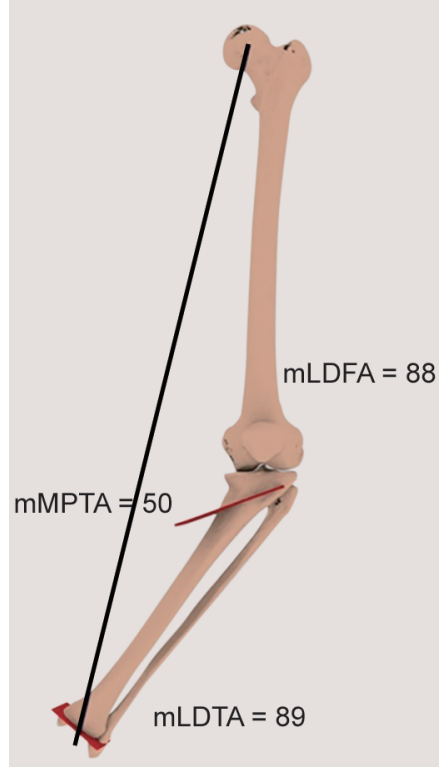

D
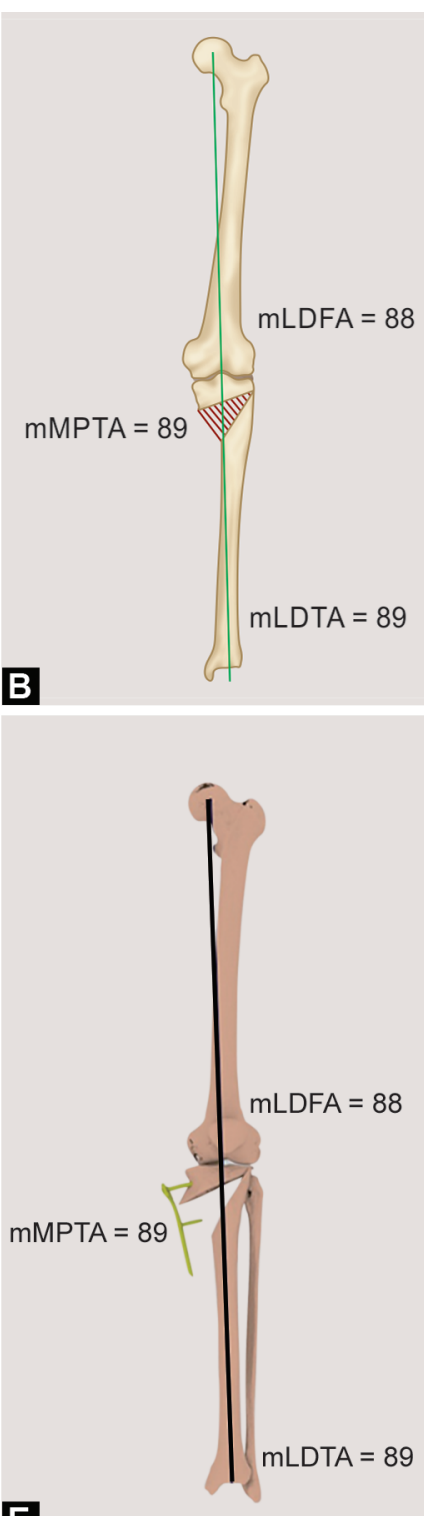

$\mathbf{E}$

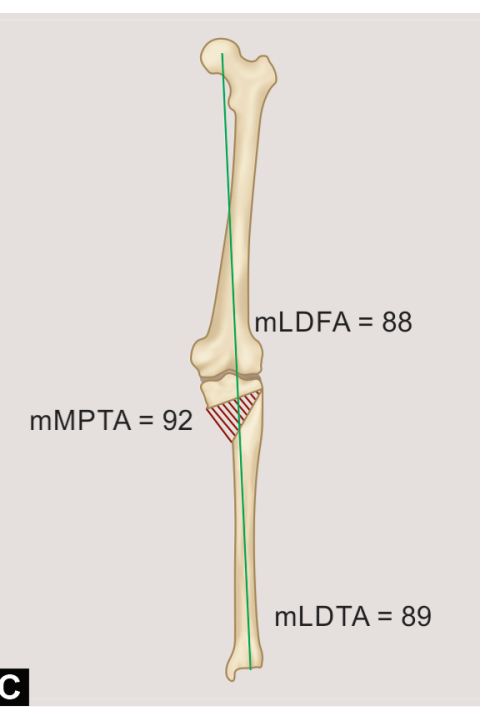

C

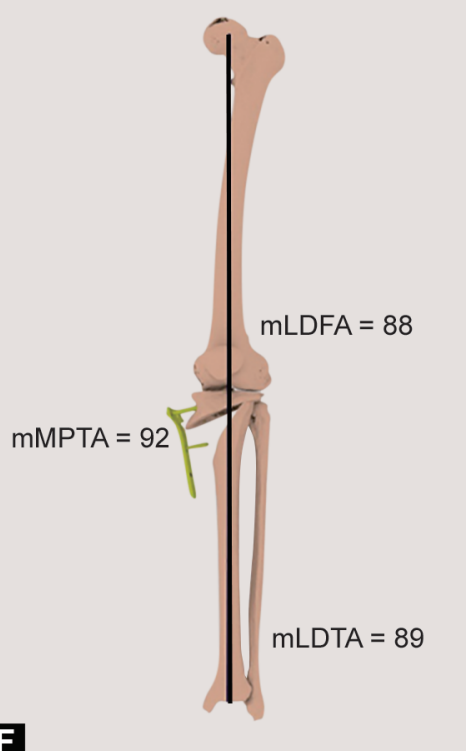

Figs $6 \mathrm{~A}$ to F: Models $\mathrm{A}$ to $\mathrm{C}$ demonstrate the technical impossibility of deformity correction and plate fixation of bone fragments with MPTA = $50^{\circ}: 2 \mathrm{D}$ simulation (A-initial deformity, $\mathrm{B}$ - correction to the neutral position of the mechanical axis, $\mathrm{C}$-overcorrection). Models $\mathrm{D}$ to $\mathrm{F}$ show the technical impossibility of deformity correction and plate fixation of bone fragments with MPTA $=50^{\circ}: 3 \mathrm{D}$ simulation (D—initial deformity, E-correction to the neutral position of the mechanical axis, F-overcorrection)

different MPTA values, the authors did not evaluate the effectiveness of correction by other types of proximal tibia osteotomies; e.g., closing-wedge, chevron and hinge type osteotomies. This study also did not investigate the relationships in tibiofibular joints before and after correction. Follow-up studies will address the above issues.

\section{Conclusion}

A comprehensive approach to preoperative planning and selection of patients (taking into account the degree and other characteristics of the deformity) would help to reduce the high level of technical and functional complications associated with high tibial osteotomy and make the outcomes of this intervention more predictable. In cases of $\mathrm{MMPTA} \geq 80^{\circ}$ where localisation of the apex of the varus deformity is at the level of the knee joint line, a proximal tibial osteotomy as described with use with proprietary medial tibial implants is acceptable. In cases of $\mathrm{mMPTA} \leq 70^{\circ}$, the distance between the lower edge of the bone plate and the medial surface of the tibia after a proximal tibial osteotomy exceeds $11 \mathrm{~mm}$ and is therefore unacceptable for use of anatomical plate designed for this location. Mechanical axis deviation to the Fujisawa point, associated with mMPTA values outside the reference range and its relevance is to be established in further studies. 


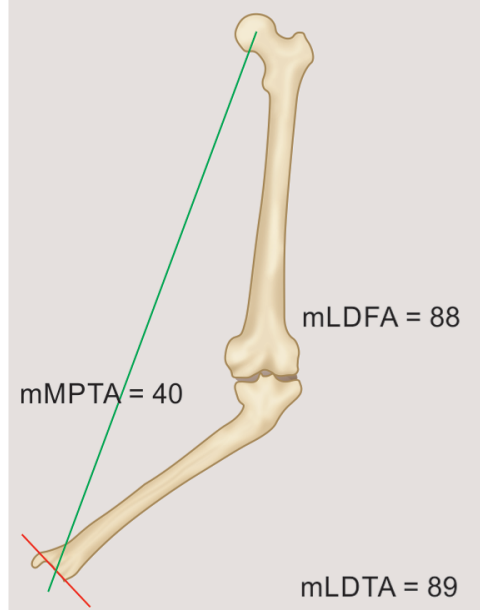

A

D

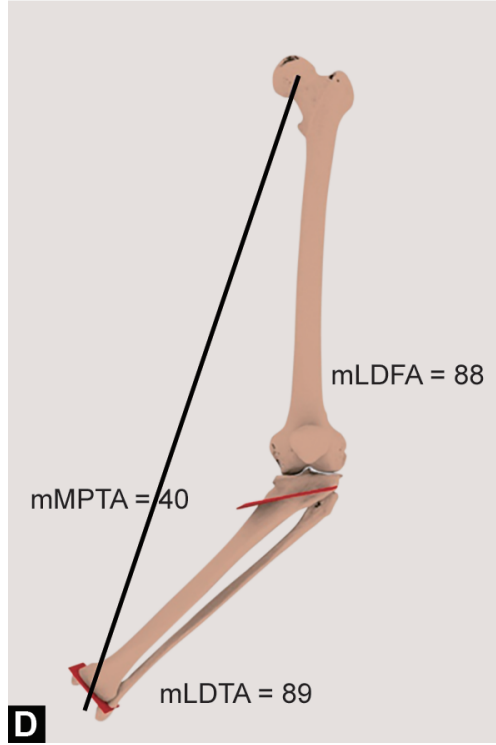

B
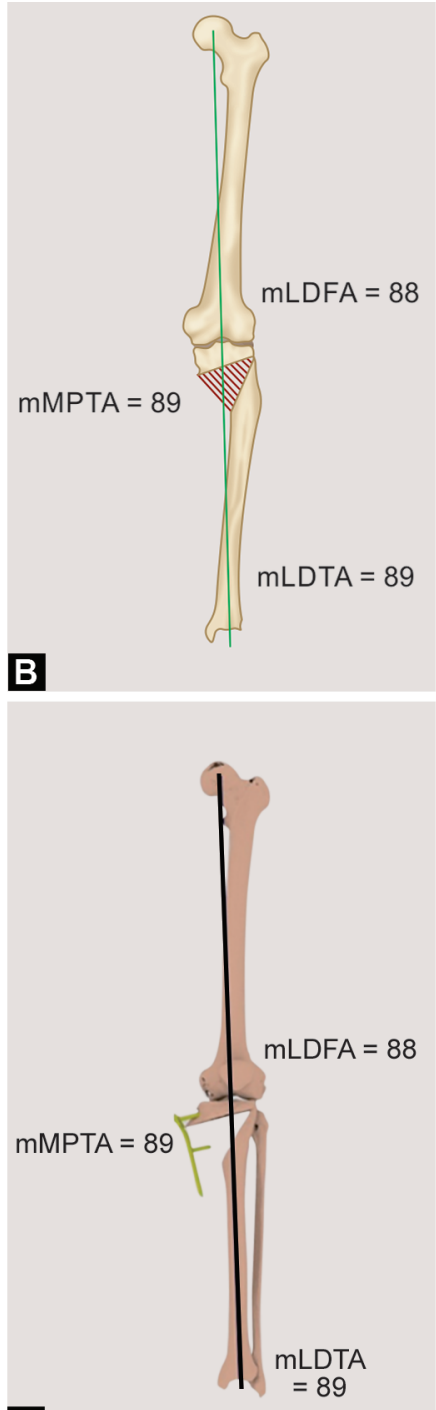

$\mathbf{E}$

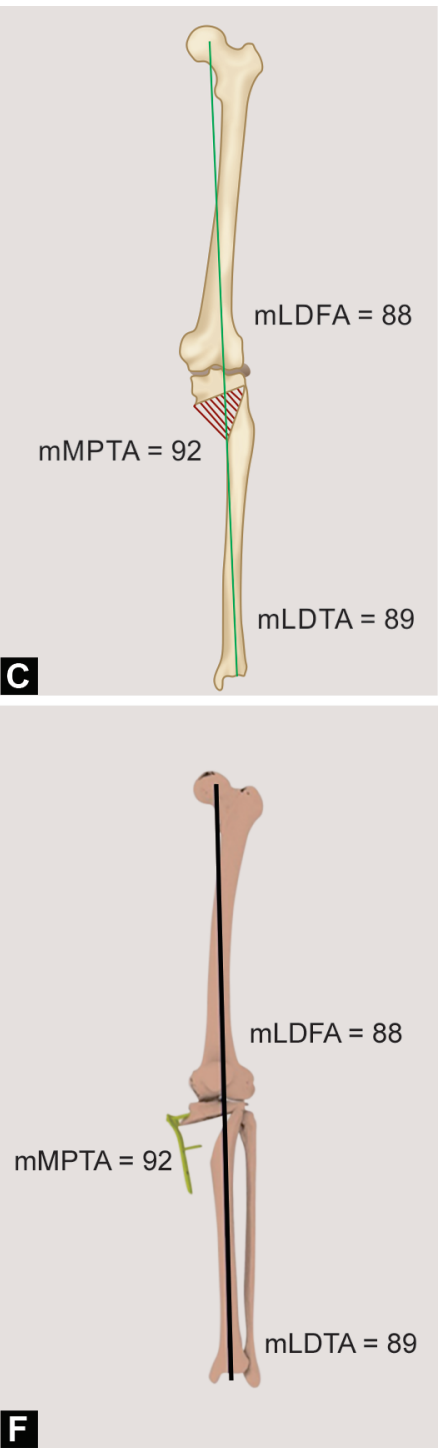

Figs 7A to F: Models A to $C$ demonstrate the technical impossibility of deformity correction and plate fixation of bone fragments with $M P T A=40^{\circ}$ : 2D simulation ( $A$-initial deformity, B-correction to the neutral position of the mechanical axis, $C$-overcorrection). Models $D$ to $F$ show the technical impossibility of deformity correction and plate fixation of bone fragments with MPTA $=40^{\circ}: 3 \mathrm{D}$ simulation (D-initial deformity, Ecorrection to the neutral position of the mechanical axis, F-overcorrection)

\section{References}

1. Jackson JP, Waugh W. Tibial osteotomy for osteoarthritis of the knee. J Bone Joint Surg Br 1961;43-B(4):746-751. DOI: 10.1302/0301620X.43B4.746.

2. Jackson JP, Waugh W, Green JP. High tibial osteotomy for osteoarthritis of the knee. J Bone Joint Surg Br 1969;51(1):88-94.

3. Liang G, Madry H, Chugaev D, et al. Advances in modern osteotomies around the knee. J Exp Orthop 2019;6(1):9. DOI: 10.1186/s40634-0190177-5.

4. Gebhard F, Krettek C, Hüfner T, et al. Reliability of computer-assisted surgery as an intraoperative ruler in navigated high tibial osteotomy. Arch Orthop Trauma Surg 2011;131(3):297-302. DOI: 10.1007/s00402010-1145-9.

5. Fujisawa Y, Masuhara K, Shiomi S. The effect of high tibial osteotomy on osteoarthritis of the knee. Orthop Clin North Am 1979;10(3): 585-608.

6. Lobenhoffer P. Stellenwert der knienahen osteotomie bei medialer gonarthrose. Orthopäde 2014;43(5):425-431. DOI: 10.1007/s00132013-2189-7.
7. Pérez-Mañanes $R$, Burró JA, Manaute JR, et al. $3 D$ surgical printing cutting guides for open-wedge high tibial osteotomy: do it yourself. J Knee Surg 2016;29(8):690-695. DOI: 10.1055/s-0036-1572412.

8. Staubli AE, De Simoni C, Babst R, et al. Tomofix: a new LCP-concept for open wedge osteotomy of the medial proximal tibia-early results in 92 cases. Injury 2003;34(2):B55-B62. DOI: 10.1016/j.injury.2003. 09.025 .

9. Harris WR, Kostuik JP. High tibial osteotomy for osteoarthritis of the knee. J Bone Joint Surg Am 1970;52(2):330-336. DOI: 10.2106/00004623-197052020-00014.

10. Insall JN. High tibial osteotomy in the treatment of osteoarthritis of the knee. Surg Annu 1975;7:347-359.

11. Salenius P, Kivilaakso R. High tibial osteotomy with Charnley's compression device. Ann Chir Gynaecol Fenn 1970;59(1):42-45.

12. Dunbar MJ, Richardson G, Robertsson O. I can't get no satisfaction after my total knee replacement: rhymes and reasons. Bone Joint J 2013;95-B(11 Suppl A):148-152. DOI: 10.1302/0301-620X.95B11.32767.

13. Kulyaba TA, Kornilov NN. Revision Knee Arthroplasty. SaintPetersburg 2016. p. 192. 
14. Hofmann S, Lobenhoffer P, Staubli A, et al. Osteotomies around the knee. European Instructional Lectures. Copenhagen, Denmark: EFORT Congress; 2011. pp. 177-189.

15. Gorecki P, Gorecki W. Jan Mikulicz-Radecki (1850-1905) - the creator of modern European surgery. Dig Surg 2002;19(4):313-318. DOI: 10.1159/000064574.

16. Paley D, Herzenberg JE, Tetsworth $\mathrm{K}$, et al. Deformity planning for frontal and sagittal plane corrective osteotomies. Orthop Clin North Am 1994;25(3):425-465.

17. Paley D. Principles of deformity correction Holly L, Anderson P, ed. Essentials of Spinal Stabilization. Springer, Cham; 2017. pp. 487-504.

18. Barksfield RC, Monsell FP. Predicting translational deformity following opening-wedge osteotomy for lower limb realignment. Strategies Trauma Limb Reconstr 2015;10(3):167-173. DOI: 10.1007/s11751-0150232-4.

19. Whitaker AT, Gesheff MG, Jauregui JJ, et al. Comparison of PACS and bone ninja mobile application for assessment of lower extremity limb length discrepancy and alignment. J Child Orthop 2016;10(5):439443. DOI: 10.1007/s11832-016-0761-5.

20. Jones GG, Jaere M, Clarke S, et al. 3D printing and high tibial osteotomy. EFORT Open Rev 2018;3(5):254-259. DOI: 10.1302/20585241.3.170075.

21. Kim HJ, Park J, Park KH, et al. Evaluation of accuracy of a threedimensional printed model in open-wedge high tibial osteotomy. J Knee Surg 2019;32(9):841-846. DOI: 10.1055/s-0038-1669901.

22. Kuriyama S, Morimoto $\mathrm{N}$, Shimoto $\mathrm{T}$, et al. Clinical efficacy of preoperative 3D planning for reducing surgical errors during openwedge high tibial osteotomy. J Orthop Res 2019;37(4):898-907. DOI: 10.1002/jor.24263.

23. Munier M, Donnez M, Ollivier M, et al. Can three-dimensional patient-specific cutting guides be used to achieve optimal correction for high tibial osteotomy? Pilot study. Orthop Traumatol Surg Res 2017;103(2):245-250. DOI: 10.1016/j.otsr.2016.11.020.

24. van Egmond N, Janssen D, Hannink G, et al. Biomechanical comparison of two different locking plates for open wedge high tibial osteotomy. J Orthop Sci 2018;23(1):105-111. DOI: 10.1016/j. jos.2017.09.014.

25. Kyung HS, Lee BJ, Kim JW, et al. Biplanar open wedge high tibial osteotomy in the medial compartment osteoarthritis of the knee joint: comparison between the Aescula and TomoFix plate. Clin Orthop Surg 2015;7(2):185-190. DOI: 10.4055/cios.2015. 7.2.185.

26. Takeuchi R, Woon-Hwa J, Ishikawa H, et al. Primary stability of different plate positions and the role of bone substitute in open wedge high tibial osteotomy. Knee 2017;24(6):1299-1306. DOI: 10.1016/j. knee.2017.07.015.

27. Nakamura R, Komatsu N, Fujita K, et al. Appropriate hinge position for prevention of unstable lateral hinge fracture in open wedge high tibial osteotomy. Bone Joint J 2017;99-B(10):1313-1318. DOl: 10.1302/0301-620X.99B10.BJJ-2017-0103.R1.

28. Ogawa $\mathrm{H}$, Matsumoto $\mathrm{K}$, Akiyama $\mathrm{H}$. The prevention of a lateral hinge fracture as a complication of a medial opening wedge high tibial osteotomy: a case control study. Bone Joint J 2017;99-B(7):887-893. DOI: 10.1302/0301-620X.99B7.BJJ-2016-0927.

29. Zhang K, Wu M. Study on lateral hinge fracture during open wedge high tibial osteotomy. Zhongguo Xiu Fu Chong Jian Wai Ke Za Zhi 2019;33(1):110-114. DOI: 10.7507/1002-1892.201807049.

30. Schröter S, Nakayama H, Yoshiya S, et al. Development of the double level osteotomy in severe varus osteoarthritis showed good outcome by preventing oblique joint line. Arch Orthop Trauma Surg 2019;139(4):519-527. DOI: 10.1007/s00402-0183068-9. 